\title{
Tumoricidal activity of combining the agonistic DR5 antibody D-6 with cisplatin in C30 cisplatin-resistant ovarian cancer in vitro and in vivo
}

\author{
YAN HUANG $^{1}$, BAOQUAN LIANG $^{1}$, QIN JIANG ${ }^{1}, \mathrm{CHEN} \mathrm{CHEN}^{1}, \mathrm{KUN} \mathrm{YANG}^{2}, \mathrm{CHUNMEI} \mathrm{LI}^{1}$ and AI ZHENG ${ }^{1}$ \\ ${ }^{1}$ Department of Obstetrics and Gynecology, West China Second University Hospital; ${ }^{2}$ Department of Gastrointestinal Surgery, \\ West China Hospital, Sichuan University, Chengdu, Sichuan 610041, P.R. China
}

Received October 15, 2013; Accepted March 19, 2014

DOI: $10.3892 / \mathrm{mmr} .2014 .2193$

\begin{abstract}
A previous study by our group reported that the agonistic DR5 antibody D-6 was capable of triggering apoptosis in A2780 cisplatin-sensitive ovarian cancer cells and that this marked effect was enhanced by cisplatin in vitro. The present study examined whether D-6 and cisplatin may exert the same anti-tumor effect on $\mathrm{C} 30$ cisplatin-resistant ovarian cancer cells, and the underlying mechanisms were investigated. D-6 exhibited an apoptosis-inducing effect, increased the cell growth inhibition rate of $\mathrm{C} 30$ cells in a dose-dependent manner, induced significant morphological changes characteristic for apoptosis, as observed by electron microscopy, and downregulated the expression of caspase 3,8 and 9 precursors in C30 cells treated with D-6 at the protein level. All of these effects were evidently enhanced when accompanied by cisplatin. Furthermore, D-6 alone or in combination with cisplatin in the established models of C30 tumor xenografts resulted in a significant repression of tumor growth, and evident apoptosis, as determined by a terminal transferase dUTP nick end labeling assay. In addition, the expression of caspase 3, 8 and 9 precursors in the tumor xenografts was as similar to that found in vitro. In conclusion, the present study suggested that D-6 may serve as a novel anti-tumor agent against C30 cisplatin-resistant ovarian cancer, with the ability to trigger apoptosis via caspase-dependent and -independent pathways and the potential to decrease the cisplatin resistance of the C30 cell line.
\end{abstract}

Correspondence to: Professor Chunmei Li or Professor Ai Zheng, Department of Obstetrics and Gynecology, West China Second University Hospital, Sichuan University, 20 People's South Road, Chengdu, Sichuan 610041, P.R. China

E-mail:1cmwch@126.com

E-mail: zazheng716@yahoo.com

Key words: TNF-related apoptosis-inducing ligand, agonistic DR5 antibody D-6, death receptors, cisplatin-resistant ovarian cancer cell line $\mathrm{C} 30$

\section{Introduction}

Ovarian cancer is one of the most lethal of all gynecological cancer types, and although its incidence is less frequent than that of cervical and endometrial cancer, it has the highest mortality rate (1). In 2010, 21,880 new cases of ovarian cancer and 13,850 mortalities were estimated in America (2). In recent decades, although there have been a number of improvements in surgical techniques and combined chemotherapeutic strategies, which are the main treatments for ovarian cancer, identifying novel effective and comprehensive treatment methods remains urgent. As one of the main, first-line chemotherapeutic treatments for ovarian cancer, cisplatin has wide clinical applications; however, cisplatin-resistance is both common and severe, posing a major challenge for improving treatment efficacy and mortality rates. Therefore, studies investigating the mechanisms of platinum-based drug resistance, new anti-tumor drugs and drug resistance-reversal agents are urgently required.

Tumor necrosis factor-related apoptosis-inducing ligand (TRAIL), also known as Apo-2 ligand (Apo2L), is a member of the tumor necrosis factor superfamily $(3,4)$. It induces apoptosis selectively in tumor cells through the caspase-dependent death signaling pathway. TRAIL binds to five receptors, including DR4 (TRAIL-R1) and DR5 (TRAIL-R2), which contain a death domain in their cytoplasmic $\mathrm{COOH}$-terminal portions and are able to induce programmed cell death (5); DcR1 (TRAIL-R3), DcR2 (TRAIL-R4) and osteoprotegrin, which act as decoy receptors without intracytoplasmic death domains, prevent cells from TRAIL-mediated apoptosis. In the majority of tumor cell types, the expression of DR5, which is rare or even absent in normal cells, is the predominant death receptor expression type of TRAIL (6) and naturally, DR5 becomes the key mediator of TRAIL-mediated cell death. Recent findings have demonstrated the defects of TRAIL, including shorter half-life in the circulatory system, cytotoxicity in human hepatocytes and normal brain tissue $(7,8)$, and TRAIL-resistance may compromise its application clinically. Therefore, to find an alternative to TRAIL for cancer treatment, the agonistic DR5 antibody, inducing apoptosis via targeting DR5, has attracted notable attention worldwide. Similar to the TRAIL-mediated apoptosis mechanism, the 
agonistic DR5 antibody may directly bind to DR5 on the cell membrane, activating caspase-dependent apoptotic pathways to induce apoptosis of target cells $(9,10)$. Additionally, these DR5 monoclonal antibodies not only had a longer half-life in vivo compared with the TRAIL ligand (11), but may have improved safety and specificity (10). Evidence of this principle has been reported with murine, rabbit or human agonistic DR5 antibodies, exhibiting a tumoricidal activity in vitro and in vivo (12).

Agonistic DR5 antibodies may also interact synergistically with chemotherapeutic agents with anti-tumor activities similar to TRAIL. Not only was it reported that several chemotherapeutic agents upregulate the mRNA expression of DR4 and DR5 via the p53-dependent or other independent signaling pathways (13-15), but these DR4 and DR5 monoclonal antibodies also enhanced the anti-tumor effects of chemotherapeutic agents (16).

A recent study on the cisplatin-resistance mechanism determined that the apoptosis-inducing effect of cisplatin on ovarian cancer mainly proceeded via the intrinsic mitochondria-dependent apoptosis pathway (17). Since the agonistic DR5 antibody markedly induced the apoptosis of several ovarian cancer cells via extrinsic caspase-dependent pathways $(9,18)$, a more effective ovarian cancer therapeutic strategy for cisplatin-resistant ovarian cancer may be examined by combination of the two different anti-tumor agents, due to their different apoptosis signaling pathways. Such a combination therapy may improve the crisis of the cisplatin-resistance in ovarian cancer treatment, change or even enhance the apoptosis-inducing effect of cisplatin on cisplatin-resistant ovarian cancer.

In the present study, the anti-tumor effect of D-6, an agonistic DR5 antibody, was investigated as a potential candidate for TRAIL, alone or accompanied with cisplatin on the C30 cisplatin-resistant ovarian cancer cell line, and the associated apoptosis mechanism was examined. Furthermore, the present study focused on whether or not D-6 would also exhibit apoptosis-inducing activity in C30 xenograft models and the difference in the anti-tumor effect of cisplatin when in combination with D-6 in C30 cisplatin-resistant ovarian cancer.

\section{Materials and methods}

Cell culture and reagents. C30 is a cisplatin-resistant ovarian cancer cell line, with a tolerance to cisplatin at a concentration of $30 \mu \mathrm{mol} / 1$, that was generously provided by Kanghong Co. (Chengdu, China). The C30 cells were cultured in RPM1-1640 culture medium containing $10 \%$ fetal bovine serum and maintained at $37^{\circ} \mathrm{C}$ in $5 \% \mathrm{CO}_{2} / 95 \%$ humidified air. The RPMI-1640 culture medium, dimethylsulfoxide (DMSO) and fetal bovine serum (FBS) were purchased from Gibco-BRL (Carlsbad, CA, USA). The 4-[3-(4-iodophenyl)-2-(4-nitrophenyl)-2H-5 -tetrazolio]-1,3-benzene disulfonate (WST-1) cell proliferation reagent kit for WST-1-based cell cytotoxicity assay was purchased from Roche Diagnostics GmbH (Mannheim, Germany). The agonistic DR5 antibody (D-6), caspase 3,8 and 9 precursor antibodies, and $\beta$-actin antibody were purchased from Santa Cruz Biotechnology, Inc. (Santa Cruz, CA, USA). Cisplatin was purchased from Gejiu Bio-Pharmaceutical Co., Ltd. (Yunnan, China).
WST-1-based cytotoxicity assay. The C30 cells were counted and cultured in two 96-well, flat bottom plates at a concentration of $5 \times 10^{4}$ cells/well and a volume of $100 \mu \mathrm{l} /$ well in 35 wells in each plate. In one plate, 30 wells were randomized into six groups (five wells/group as five replicates) and to every well, $100 \mu \mathrm{l}$ RPMI-1640 culture medium $+10 \%$ FBS, containing various amounts of D-6 (final concentration, e.g. 0.125, 0.25, 0.5, 1,2 and $4 \mu \mathrm{g} / \mathrm{ml}$ ) was added, while the remaining five wells as the control group were cultured in medium only. The other plate was treated as the above one, except for that cisplatin $(2.5 \mu \mathrm{g} / \mathrm{ml})$ was added to every well with the six serial concentrations of D-6. Following $48 \mathrm{~h}$ culture of the cells at $37^{\circ} \mathrm{C}$ in $5 \% \mathrm{CO}_{2} / 95 \%$ humidified air, $10 \mu \mathrm{l}$ WST-1 was added to each well. The plates were then incubated for an additional $4 \mathrm{~h}$. The plates were then thoroughly agitated for $1 \mathrm{~min}$, and the absorbance values of the sample in each well were measured at $440 \mathrm{~nm}$ by a Varioskan Flash reader (Thermo Fisher Scientific, Waltham, MA, USA). The cell growth inhibition rate was calculated as follows: [1-(OD test-OD blank)/(OD control-OD blank)] x 100\% (19).

Western blot analyses. The C30 cells were counted and cultured in $25 \mathrm{~cm}^{2}$ culture flasks at a concentration of $1 \times 10^{6}$ cells/flask. Following $12 \mathrm{~h}$ of culture in the incubator, the supernatant was extracted and the four flasks were randomized into four groups: The control group, where $5 \mathrm{ml}$ culture medium was added; the cisplatin group, where $5 \mathrm{ml}$ culture medium containing $2.5 \mu \mathrm{gml}$ cisplatin was added; the D- 6 group, where $5 \mathrm{ml}$ culture medium containing $2 \mu \mathrm{g} / \mathrm{ml} \mathrm{D}-6$ was added; the combination group, where $5 \mathrm{ml}$ culture medium containing $2.5 \mu \mathrm{gml}$ cisplatin and $2 \mu \mathrm{g} / \mathrm{ml} \mathrm{D}-6$ was added. Following $48 \mathrm{~h}$ in the incubator, the cells from each group were collected into four different tubes and then protein of the cells was extracted using a Total Protein Extraction kit (Millipore Chemicon, MA, USA), and then determined with a Bradford Protein Assay kit (Bio-Rad, Hercules, CA, USA) and measured at $595 \mu \mathrm{m}$ using a Varioskan Flash reader (Thermo Fisher Scientific). Similarly, the xenograft tumors from the four different groups were homogenized using a technical homogenizer and protein extraction was performed as for the cells. The protein (40 $\mu$ gwell) extracted from the cells or tissues of each group was subjected to SDS-PAGE for $30 \mathrm{~min}$ at $80 \mathrm{~V}$, followed by $50 \mathrm{~min}$ at $110 \mathrm{~V}$. Then, the gels were electroblotted on polyvinylidene fluride membranes (Bio$\mathrm{Rad}$ ) for $1.5 \mathrm{~h}$ at $250 \mathrm{~mA}$. The membranes were subsequently incubated in 5\% non-fat milk powder in Tris-buffered saline (TBS) containing $0.05 \%$ Tween-20 (TBS-T) for $1-2 \mathrm{~h}$ to block non-specific binding sites and then incubated in the appropriate primary antibody overnight. The concentration of these primary antibodies were as follows: Procaspase-3 (dilution, 1:250), procaspase-8 (dilution, 1:500), procaspase-9 (dilution, 1:500) and $\beta$-actin (dilution, 1:1,000). The following morning, the membranes were incubated at room temperature for $1 \mathrm{~h}$ in secondary antibody (Zsgb-bio, Beijing, China), after rinsing three times in TBS-T. Finally, the membranes were incubated with enhanced chemoluminescence western blotting detection reagent (Bio-Rad) for 1-2 min and exposed to a ChemiDoc XRS system (Bio-Rad).

Electron microscopic analysis of morphological changes during apoptosis. The C30 cells from the four different groups were treated as described above in the western blot analyses. 
Following $24 \mathrm{~h}$ in the incubator, the cells from each group were collected into four different centrifuge tubes, washed in phosphate-buffered saline (PBS; $\mathrm{pH} 7.4$ ) and then carefully transferred into $0.5 \%$ glutaraldehyde using a pipette. Then, they were incubated for $10 \mathrm{~min}$ at $4^{\circ} \mathrm{C}$. Subsequently, the samples were fixed in $3 \%$ glutaraldehyde and fixed again with $1 \%$ osmiumtetroxide. The fixed samples were dehydrated with a gradient series of acetone and then embedded in Epon-812 agar (Shell Chemicals, Deer Park, TX, USA). Finally, the embedded samples, which were constructed into ultrathin sections by automatic semi-thin rotary microtome (Leica, Wetzlar, Germany) and stained with uranyl acetate and lead citrate, were observed under a Hitachi H-600IV transmission electron microscope (Hitachi, Tokyo, Japan) and images were also captured by the transmission electron microscope.

Creating C30 cisplatin-resistant ovarian cancer xenografts in vivo. All animal procedures were approved by the Animal Care and Scientific Committee of Sichuan University (Chengdu, China). A total of 24 4-5-week old female BALB/C (nu/nu) nude mice were purchased from the Experimental Animal Center of Sichuan University (Chengdu, China) and then raised in a specific pathogen-free animal house. Every nude mouse was inoculated subcutaneously with C30 cells $\left(1 \times 10^{7}\right)$. When the xenograft tumor volume reached $\sim 100 \mathrm{~mm}^{3}$, the nude mice were randomized into four groups. Each group contained six nude mice and was subject to the following treatment: The control group with normal saline (300 $\mu \mathrm{l} /$ mouse); the cisplatin group with cisplatin $(3 \mathrm{mg} / \mathrm{kg})$; the D-6 group with D-6 (3 mg/kg); the combination group with cisplatin ( $3 \mathrm{mg} / \mathrm{kg}$ ) and D-6 (3 mg/kg). Administration by abdominal subcutaneous injection was twice a week for 2 weeks, and the nude mice were sacrificed three days following the last administration. During the treatment time, the weight of the animals and the xenograft tumor volume were measured at the time-point of each administration and at the termination of the study. The xenograft tumor volume was measured with a vernier caliper and calculated by the product of the maximum transverse diameter, the maximum width and height. The xenograft tumor of each nude mouse, which was completely stripped, was divided into two parts; one was stored in $4 \%$ paraformaldehyde and the other was snap-frozen in liquid nitrogen for several subsequent assays.

Terminal transferase dUTP nick end labeling (TUNEL) assay. Apoptosis in the xenograft tumors from nude mice was detected by an in situ cell death detection kit, alkaline phosphatase (AP) (Roche Diagnostics $\mathrm{GmbH}$ ) according to manufacturer's instructions. The xenograft tumors, immersed in $4 \%$ polyformaldehyde, were embedded in paraffin, and then dewaxed with xylene twice for $10 \mathrm{~min}$ and alcohol at 100,90 and $80 \%$ concentration once for $2 \mathrm{~min}$. The sections were subsequently digested with proteinase $\mathrm{K}(20 \mu \mathrm{g} / \mathrm{ml})$ for $20 \mathrm{~min}$, rinsed with distilled water, treated in 3\% hydrogen peroxide for $10 \mathrm{~min}$, rinsed with distilled water again and PBS twice for $5 \mathrm{~min}$. The TUNEL reaction mixture (1:10 dilution; Roche Diagnostics $\mathrm{GmbH}$ ) was added dropwise to the sections at $30 \mu \mathrm{l} /$ section which were then maintained at a constant temperature of $37^{\circ} \mathrm{C}$ for $1 \mathrm{~h}$. Following rinsing with PBS twice for $3 \mathrm{~min}$, anti-fluorescein isothiocyanate (FITC)-AP

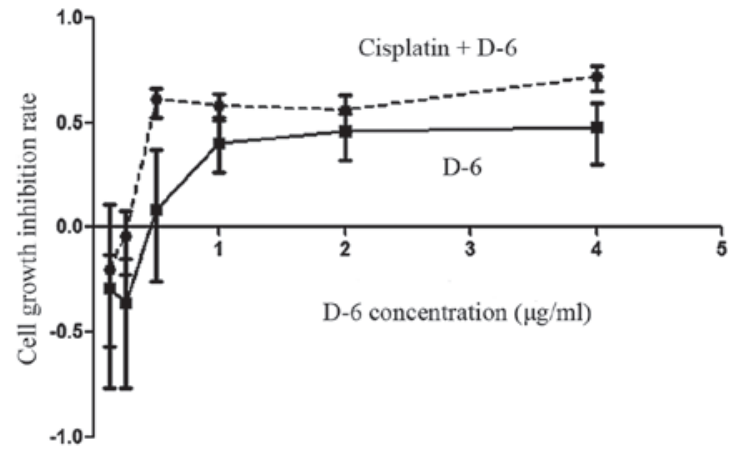

Figure 1. Cell growth inhibition rate of C30 cisplatin-resistant ovarian cancer cells analyzed by WST-1-based cytotoxicity assay. (----) Combination group: Cells were treated with various concentrations $(0.125,0.25,0.5,1$, 2 and $4 \mu \mathrm{g} / \mathrm{ml})$ of D-6 and cisplatin at a constant concentration $(2.5 \mu \mathrm{g} / \mathrm{ml})$. (-_-) D-6 group: Cells were treated with various concentrations $(0.125$ $0.25,0.5,1,2$ and $4 \mu \mathrm{g} / \mathrm{ml}$ ) of D-6. WST-1, 4-[3-(4-iodophenyl)-2-(4-nitroph enyl)-2H-5-tetrazolio]-1,3-benzene disulfonate.

(ready-to-use) was added dropwise at 20-30 $\mu \mathrm{l} / \mathrm{section}$ and sections were maintained at a constant temperature of $37^{\circ} \mathrm{C}$ for $30 \mathrm{~min}$. Finally, following rinsing with PBS twice for $3 \mathrm{~min}$, the sections were developed with AP-red and observed under a light microscope (Nikon, Tokyo, Japan). Apoptotic cells stained red in the sections.

Statistical analyses. Values are expressed as the mean \pm standard deviation. The differences in cell growth inhibition rates, xenograft tumor volumes and wet weights, and apoptosis rates of xenograft tumors among the four groups, were analyzed by one-way analysis of variance. All statistical analyses were performed by the SPSS 17.0 software package (SPSS, Inc., Chicago, IL, USA) and $\mathrm{P}<0.05$ was considered to indicate a statistically significant difference.

\section{Results}

Cell growth inhibition rate. The $\mathrm{C} 30$ cell growth inhibition rates were examined by an WST-1-based cytotoxicity assay. As shown in Fig. 1, treatment of C30 cells with D-6 alone or in combination with cisplatin produced dose-dependent cell growth inhibition rates for D-6 concentrations $>0.25 \mu \mathrm{g} / \mathrm{ml}$ $(\mathrm{P}<0.05)$. These rates were more significant in the combination group than in the D-6 alone group $(\mathrm{P}<0.05)$. However, the cell growth inhibition rates decreased following treatment with D-6 concentrations of $<0.25 \mu \mathrm{g} / \mathrm{ml}$ in the D-6 group.

Caspase cascade changes in vitro. As TRAIL activates caspases in various tumor cells, it was investigated whether the agonistic DR5 antibody D-6 would also activate these apoptotic reactions. In Fig. 2, $48 \mathrm{~h}$ following treatment, the expression of caspase 3, 8 and 9 precursors in C30 cells was evidently decreased in the D-6 and combination groups compared with the control and cisplatin groups by western blot analysis, and this decrease was more evident in the combination group. The downregulation of caspase 3,8 and 9 precursors results in the upregulation of caspase 3,8 and 9, which are key factors in the caspase-dependent and -independent death signaling pathway. The results suggested that cisplatin induced weak or no 


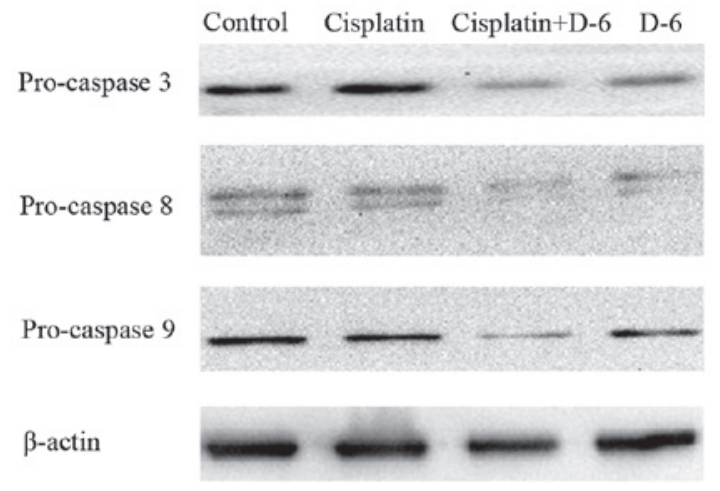

Figure 2. Western blot analyses of caspase 3, 8, 9 precursor and $\beta$-actin expression of $\mathrm{C} 30$ cells in the four different groups in vitro.

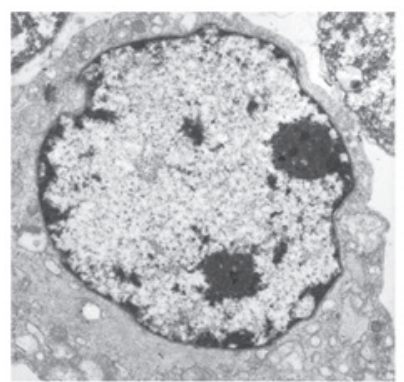

Control

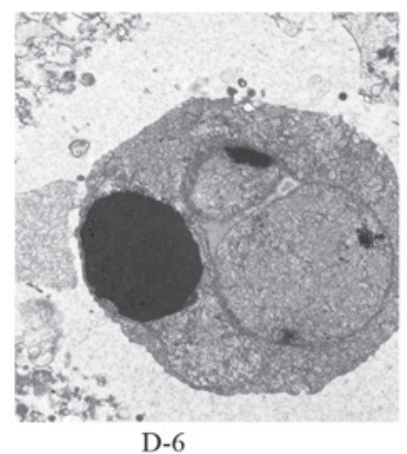

D-6

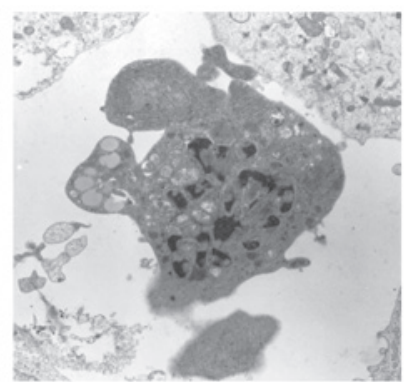

Cisplatin

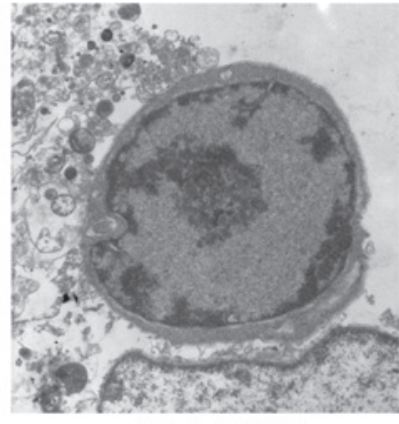

Cisplatin+D-6
Figure 3. Morphological changes in C30 cells with different treatment as observed under electron microscopy (magnification, $\mathrm{x} 8,000$ ). The control group represents the normal C30 cisplatin-resistant ovarian cancer cells. In the cisplatin group, $\mathrm{C} 30$ cells exhibited chromatin condensation and evident organelle swelling. In both the D-6 and combination groups, C30 cells exhibited nuclear pycnosis, chromatin condensation and marginalization.

cleavage of caspase 3, 8 and 9, whereas D-6 triggered marked caspase activation, with a substantially enhanced cleavage of caspase 3,8 and 9 when accompanied with cisplatin in vitro.

Morphological changes during apoptosis. As demonstrated in Fig. 3, $24 \mathrm{~h}$ following treatment, the C30 cells in the control group demonstrated a normal morphology, without any damage or significant morphological-apoptotic changes, as observed using electron microscopy. Cisplatin-only treatment resulted in mild chromatin condensation and organelle swelling in the C30 cells. By contrast, the C30 cells from the D- 6 and combination groups demonstrated morphological changes characteristic for apoptosis, including nucleus pycnosis, significant chromatin condensation and marginalization, cell

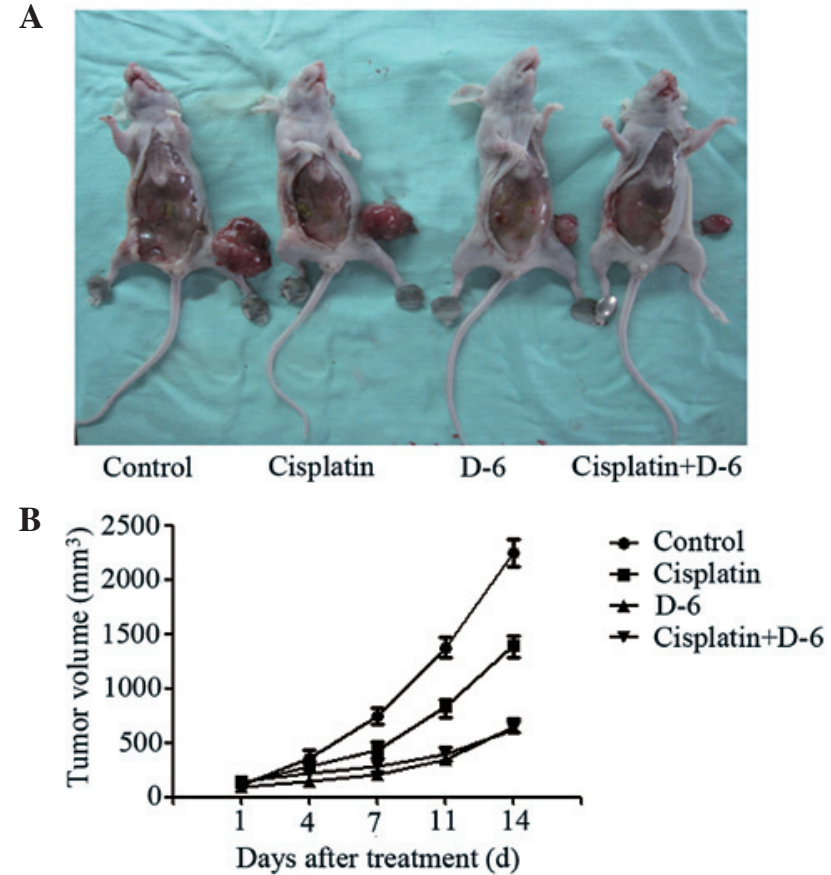

Figure 4. Anti-tumor effect of D-6 in vivo. (A) Images of xenograft tumors in nude mice following sacrifice, depicting the differences in volume between the four groups. (B) Growth curves of xenograft tumors in nude mice in each group. The vertical axis resembles the average xenograft tumor size $\left(\mathrm{mm}^{3}\right)$ and horizonal axis represents five time-points of measurement, respectively on day $1,4,7,11$ and 14 .

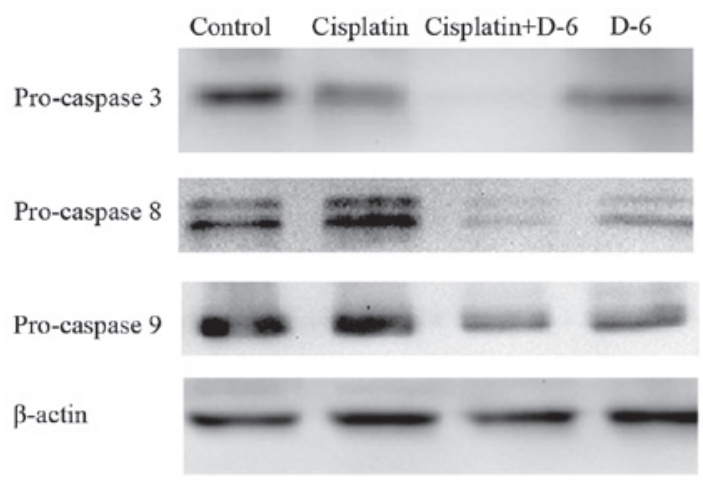

Figure 5. Western blot analyses of caspase 3, 8, 9 precursor and $\beta$-actin expression of xenograft tumors in nude mice from the four different groups in vivo.

membrane integrity, reduction in cell volume and the appearance of typical apoptotic bodies.

Anti-tumor effect of D-6 and cisplatin in xenograft models. To examine the anti-tumor activity of D-6 in vivo, the present study investigated its efficacy in xenograft models, in which C30 cells were inoculated into nude mice subcutaneously. Xenograft tumors were established at a mean volume of $100 \mathrm{~mm}^{3}$ prior to initiating the administration. For each group, the xenograft tumor volumes were measured prior to each administration and before the mice were sacrificed (Table I). The tumors grew rapidly in the control group, whereas the tumors in the other three groups advanced significant slower and demonstrated delayed trends in progression. As compared 
Table I. Summary of xenograft tumor volume in each group $\left(\mathrm{mm}^{3}\right)$.

\begin{tabular}{lcccc}
\hline Administration time (days) & Control group & Cisplatin group & D-6 group & Cisplatin + D-6 group $^{\mathrm{a}}$ \\
\hline 1 & $112.7 \pm 56.3$ & $136.4 \pm 73.1$ & $99.7 \pm 54.1$ & $145.95 \pm 48.2$ \\
4 & $362.2 \pm 215.2$ & $278.1 \pm 157.2$ & $139.8 \pm 81.8$ & $215.31 \pm 90.4$ \\
7 & $748.2 \pm 633.1$ & $427.1 \pm 266.1$ & $210.1 \pm 127.1$ & $286.53 \pm 173.4$ \\
11 & $1376.8 \pm 939.3$ & $832.7 \pm 625.4$ & $340.7 \pm 210.0$ & $395.5 \pm 264.8$ \\
14 & $2243.8 \pm 935.6$ & $1401.0 \pm 995.7$ & $650.9 \pm 295.6$ & $617.6 \pm 370.5$ \\
\hline
\end{tabular}

${ }^{\text {a }} \mathrm{P}<0.05$, compared with the control group. All results are expressed as the mean \pm standard deviation.

Table II. Summary of xenograft tumor wet weight in each group (g).

\begin{tabular}{lcccc}
\hline & Control group & Cisplatin group & D-6 group & Cisplatin+ D-6 group $^{\mathrm{a}}$ \\
\hline Wet weight & $1.35 \pm 0.7$ & $0.91 \pm 0.62$ & $0.38 \pm 0.19$ & $0.27 \pm 0.11$ \\
\hline
\end{tabular}

${ }^{a} \mathrm{P}<0.05$, compared with the control group. All results are expressed as the mean \pm standard deviation.

A

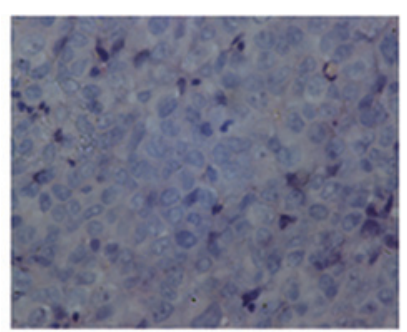

Control

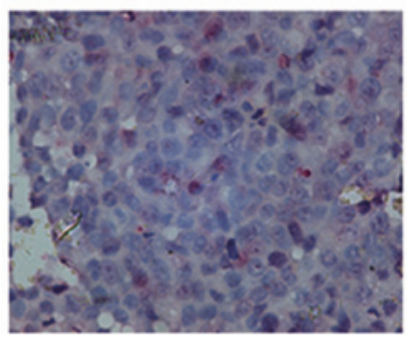

D-6

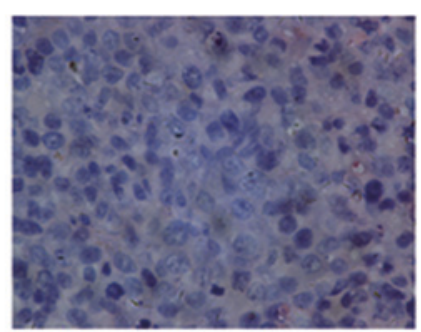

Cisplatin

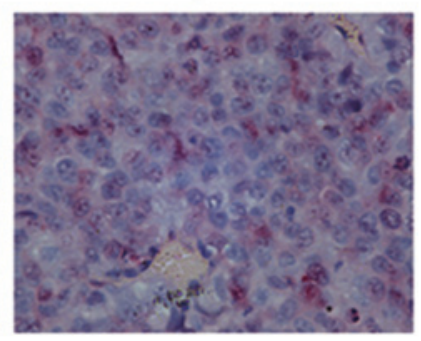

Cisplatin+D-6

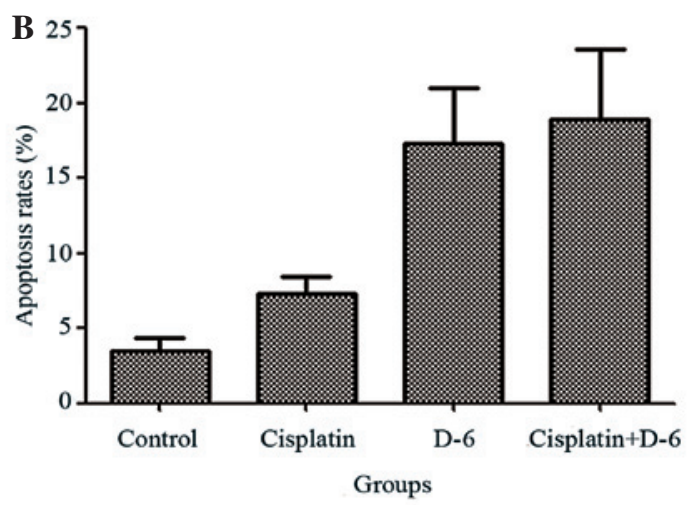

Figure 6. Immunohistochemical assessment of apoptosis by TUNEL staining (TUNEL-positive, apoptotic cells stain red) (A) Images of TUNEL-stained C30 xenograft tumor paraffin sections (magnification, $\mathrm{x} 400$ ). (B) Quantification of the images, with the rates of apoptosis being as follows: Control group, 3.54 $\pm 0.84 \%$; cisplatin group, $7.32 \pm 1.11 \%$; D-6 group, $17.32 \pm 3.67 \%$ and combination group, $18.91 \pm 4.68 \%$. TUNEL, terminal transferase dUTP nick end labeling.

with the control group, the inhibition rates (i.e. the control group volume minus the treated group volume/the control group volume at the last measured time-point) in the cisplatin, D-6 and combination groups were 37.56, 70.99 and $72.47 \%$ respectively. As shown in Fig. 4B, the administration of D-6 alone or in combination with cisplatin resulted in the significant repression of tumor growth $(\mathrm{P}<0.05)$, and the evident gross change of the xenograft tumor volume is also illustrated in Fig. 4A. However, the xenograft tumors in the combination group demonstrated higher inhibition rates and smaller tumor volume compared with the D-6 group with no significance.

Following sacrificing the mice, the entire xenograft tumors were completely removed and weighed and the results of the wet weights are illustrated in Table II. The tumor wet weights in the D-6 and combination groups were the smallest among the four groups, consistent with the changes of the tumor volumes.

Caspase cascade changes in vivo. Following sacrificing the xenograft mice, western blot analyses were performed on the xenograft tumors to detect changes in the expression of the caspase precursors. The expression of caspase 3,8 and 9 precursors changed in accordance with the results in C30 cells following $48 \mathrm{~h}$ treatment in vitro (Fig. 5). D-6 induced the cleavage of caspase 3,8 and 9 , and when in combination with cisplatin, caused a markedly greater caspase activation. 
However, weak or no caspase activation reactions occurred in the cisplatin-treated xenograft tumors.

Assessment of apoptosis by TUNEL staining. TUNEL-positive cells, stained red, were observed in the xenograft tumor sections among the four different groups. As shown in Fig. 6A, there were notably more red stained cells in the D-6 and the combination groups as compared with the cisplatin and control groups. The rates of apoptosis of the four groups are illustrated in Fig. 6B, demonstrating that D-6 induced substantial apoptosis, with the rate of apoptosis being $17.32 \pm 3.67 \%$, and this rate was increased in the combination group with a rate of $18.91 \pm 4.68 \%$. The increases in rates of apoptosis in the D-6 and combination groups compared with those of the control and the cisplatin groups were statistically significant $(\mathrm{P}<0.05)$.

\section{Discussion}

Ovarian cancer is associated with poor prognosis and represents a severe threat to female reproductive health and quality of life. A large number of patients with ovarian cancer present in the advanced ovarian cancer stages at first diagnosis. As a result, developing effective treatment strategies to improve the prognosis of ovarian cancer is highly important. The majority of ovarian cancer cases are sensitive to platinum-based chemotherapy in the initial treatment stages; however, later recurrence of the disease, as is common for most patients, is associated with the development of platinum resistance (18). Therefore, the identification of novel anti-tumor drugs and drug resistance-reversal agents is urgently required. TRAIL/TRAIL receptors, as members of the tumor necrosis factor superfamily, have an important role in regulating apoptosis of malignant neoplasms. They exhibit notable anti-tumor effects via activation of caspase-dependent and -independent pathways $(10,12)$, which is an effect that is enhanced by exposure to chemotherapy, including cisplatin, adriamycin, BisVIII and others $(20,21)$. The present study investigated the in vitro tumoricidal activity of an agonistic antibody to DR5, D-6, alone or in combination with cisplatin, against the $\mathrm{C} 30$ cisplatin-resistant ovarian cancer cell line, which is resistant to cisplatin at a high concentration of $30 \mu \mathrm{mol} / \mathrm{l}$. Additionally, xenograft models based on C30 cells were established to further examine these effects in vivo. It was identified that D-6 not only induced apoptosis in tumor cells in vitro and inhibited cisplatin-resistant ovarian tumor growth in nude mice, but also enhanced the activity of cisplatin in the platinum-resistant cancer cell line.

Over the past several decades, numerous in-depth studies have investigated the efficacy and mechanisms underlying TRAIL-based cancer therapies. While the TRAIL-inducing apoptosis pathways are more thoroughly understood, its various shortcomings appear to be restricting its clinical application. Agonistic antibodies against DR4 or DR5, as potent candidates for TRAIL, have been produced in recent years, including TRA-8, CS-1008, apomab and HGS-ETR1 (12,22-25). These antibodies generally have a longer plasma half-life, increased anti-tumor activity without decoy receptors, specificity without exogenous cross-linking, and the ability to overcome TRAIL-associated cytotoxicity and resistance. Among these antibodies, the murine and human monoclonal antibodies targeting DR5 have exerted marked tumoricidal activity in vitro and in vivo $(9,25-27)$. The human monoclonal antibody conatumumab (AMG655) has been investigated in phase II clinical trials in patients with metastatic pancreatic cancer, demonstrating marked efficacy in improving the six-month survival rate and overall survival (28). D-6, a murine agonistic antibody to DR5, which was investigated in our previous study, exhibited strong anti-tumor efficacy towards the cisplatin-sensitive ovarian cancer cell line A2780 in vitro, with an enhanced effect in combination with cisplatin (9). In the present study, it was demonstrated that D-6 was also effective in triggering apoptosis of the cisplatin-resistant ovarian cancer cell line $\mathrm{C} 30$ in vitro and this effect was improved when in combination with cisplatin. Under an electron microscope, significant and typical morphological apoptosis changes were observed in the C30 cells treated with D-6, and the most evident programed cell death changes were found in the C30 cells treated with D-6 plus cisplatin. These results suggested that D-6 and cisplatin acted synergistically to induce apoptosis in ovarian cancer cells.

The results of the WST-1-based cytotoxicity assay in vitro suggested that D-6 inhibited the growth of C30 cells in a dose-dependent manner, and this inhibitory effect was enhanced by cisplatin. Studies of other agonistic DR5 antibodies in combination with chemotherapy on other tumor cells have revealed a correlation between agonistic DR5 antibodies and cisplatin (18,27). Similar to TRAIL, numerous TRAIL receptors induce apoptosis via the cell-extrinsic pathway, involving the recruitment and activation of caspase 8 , followed by ligand binding to DR4 or DR5. Of note, several recent studies also reported that a number of the TRAIL receptors not only induce caspase-dependent cell death, but also regulate a caspase-independent cell death mechanism $(10,12)$. Concurrently, numerous studies have demonstrated that the majority of chemotherapeutic agents trigger apoptosis through the cell-intrinsic pathway, by activating pro-apoptotic B-cell lymphoma 2 family members, releasing cytochrome $\mathrm{C}$ and apoptotic protease activating factor 1 , and thereafter activating caspase 9 (29). Although caspase 8 and 9 are activated by means of two independent pathways, in turn, they activate the same downstream effectors, including caspase 3,6 and 7, and subsequently stimulate programmed cell death. In the present study, the internal reaction in the $\mathrm{C} 30$ cisplatin-resistant ovarian cancer cells following treatment was examined by investigating the expression of caspase 3, 8 and 9 precursors via western blot analysis. D-6 alone or in combination with cisplatin resulted in caspase cascade expression changes in C30 cells, substantially downregulating the caspase 3,8 and 9 precursors and subsequently enhancing the activation of the associated caspases (i.e., highly activating the associated caspases, including caspase 3,8 and 9). The data suggested that the apoptosis of C30 cells in the present study occurred in a caspase-dependent and -independent manner. Cisplatin alone induced weak or no activation of caspase 3,8 and 9; however, D-6 triggered detectable processing of caspase 3,8 and 9, and enhanced cleavage of these caspases when in combination with cisplatin.

TRAIL/TRAIL receptors exhibit tumoricidual efficacy in xenograft models, consistent with in vitro evidence. The agonistic DR5 antibodies, including AD5-10 and Apomab, exhibited a marked anti-tumor effect on xenograft models 
based on a variety of tumor cell types $(10,25)$. During these investigations, it was demonstrated that the agonistic DR5 antibodies they had examined exhibited the capability to inhibit tumor growth and even regress xenograft tumor types, including lung, liver and breast cancer $(10,25,30)$. In the present study, it was demonstrated that the volume of the xenograft tumors in nude mice increased by 19.9-fold two weeks following subcutaneous injection in the control group. This aggressive tumor growth was inhibited by D-6 alone or in combination with cisplatin, and combination treatment demonstrated a more evident tumor growth repression than D-6 alone, although this difference was no statistically significant. Indeed, both the D-6 and combination groups reached a high tumor inhibition rate of $>70 \%$. Similar to the changes of tumor volume, the tumor wet weights in the D-6 and combination groups were significantly lower than those in the other two groups. Taken together, D-6 repressed the growth of xenograft tumors in nude mice, as demonstrated by the evident inhibition of the increases in both the tumor volume and wet weight. The internal reaction proteins in the processes of apoptosis in vivo were also detected, with the activation of caspase 3,8 and 9. Caspase-dependent and -independent apoptosis occurred both in vitro and in vivo, further providing evidence suggesting a mechanism of apoptosis-induction by D-6. Additionally, in order to observe the macroscopic apoptotic cells in xenograft tumor sections, apoptosis analyses were conducted by a TUNEL assay. The results demonstrated there were more macroscopic apoptotic cells in the D-6 and combination treatment groups as compared with the other two groups, which further confirmed the marked anti-tumor effect of D-6 in xenograft tumors. It was hypothesized that D-6 had a role in C30 growth inhibition and was able to trigger apoptosis in C30 cisplatin-resistant ovarian cancer via regulating the relative caspases. Furthermore, D-6 treatment in combination with cisplatin activated more caspases (3,8 and 9) than D-6 or cisplatin treatment alone. Therefore, D- 6 may be able to overcome the cisplatin-resistance in C30 cisplatin-resistant ovarian cancer by changing the apoptosis signaling pathways of cisplatin, and exerting a synergistic function on apoptosis in combination with cisplatin. However, further studies are required to elucidate the underlying synergitistic mechanisms of apoptosis induction by DR5 monoclonal antibodies with chemotherapeutic agents in drug-resistant cancer types.

In conclusion, the agonistic DR5 antibody D-6 is a potential anti-tumor agent that triggers apoptosis via caspase-dependent and -independent pathways in $\mathrm{C} 30$ cisplatin-resistant ovarian cancer. Furthermore, the reported anti-tumor effect of DR5 may be enhanced when concomitantly treated with the chemotherapeutic agent cisplatin. These results suggested that D-6 may represent a novel candidate for overcoming the cisplatin-resistance of the cell line $\mathrm{C} 30$. Therefore, these preclinical data may facilitate further large-scale clinical investigations for the development of novel therapeutic strategies for the treatment of cisplatin-resistant ovarian cancer.

\section{Acknowledgements}

The present study was supported by grants from the National Natural Scientific Foundation of China (no. 30973192).

\section{References}

1. Fialka I, Pasquali C, Kurzbauer R, Lottspeich F and Huber LA: Loss of epithelial polarity is accompanied by differential association of proteins with intracellular membranes. Electrophoresis 20: 331-343, 1999.

2. Jemal A, Siegel R, Xu J and Ward E: Cancer statistics, 2010. CA Cancer J Clin 60: 277-300, 2010.

3. Pitti RM, Marsters SA, Ruppert S, Donahue CJ, Moore A and Ashkenazi A: Induction of apoptosis by Apo-2 ligand, a new member of the tumor necrosis factor cytokine family. J Biol Chem 271: 12687-12690, 1996.

4. Wiley SR, Schooley K, Smolak PJ, et al: Identification and characterization of a new member of the TNF family that induces apoptosis. Immunity 3: 673-682, 1995.

5. Lacour S, Hammann A, Wotawa A, Corcos L, Solary E and Dimanche-Boitrel MT: Anticancer agents sensitize tumor cells to tumor necrosis factor-related apoptosis-inducing ligand-mediated caspase-8 activation and apoptosis. Cancer Res 61: 1645-1651, 2001.

6. Ma Y, Yang D and Chen Y: Analysis of TRAIL receptor expression using anti-TRAIL death receptor-5 monoclonal antibodies. Chin Med J (Engl) 116: 947-950, 2003.

7. Jo M, Kim TH, Seol DW, et al: Apoptosis induced in normal human hepatocytes by tumor necrosis factor-related apoptosis-inducing ligand. Nat Med 6: 564-567, 2000.

8. Nitsch R, Bechmann I, Deisz RA, et al: Human brain-cell death induced by tumour-necrosis-factor-related apoptosis-inducing ligand (TRAIL). Lancet 356: 827-828, 2000.

9. Jiang Q, Zhu H, Liang B, Huang Y and Li C: Apoptosis-inducing effect of the DR5 monoclonal antibody, D-6, alone or in combination with cisplatin, on A2780 ovarian cancer cells. Mol Med Rep 6: 316-320, 2012.

10. Guo Y, Chen C, Zheng Y, et al: A novel anti-human DR5 monoclonal antibody with tumoricidal activity induces caspase-dependent and caspase-independent cell death. J Biol Chem 280: 41940-41952, 2005.

11. Yagita H, Takeda K, Hayakawa Y, Smyth MJ and Okumura K: TRAIL and its receptors as targets for cancer therapy. Cancer Sci 95: 777-783, 2004

12. Pukac L, Kanakaraj P, Humphreys R, et al: HGS-ETR1, a fully human TRAIL-receptor 1 monoclonal antibody, induces cell death in multiple tumour types in vitro and in vivo. Br J Cancer 92: 1430-1441, 2005.

13. Gibson SB, Oyer R, Spalding AC, Anderson SM and Johnson GL: Increased expression of death receptors 4 and 5 synergizes the apoptosis response to combined treatment with etoposide and TRAIL. Mol Cell Biol 20: 205-212, 2000.

14. Wu GS, Burns TF, McDonald ER III, et al: KILLER/DR5 is a DNA damage-inducible p53-regulated death receptor gene. Nat Genet 17: 141-143, 1997.

15. Sheikh MS, Burns TF, Huang Y, et al: p53-dependent and -independent regulation of the death receptor KILLER/DR5 gene expression in response to genotoxic stress and tumor necrosis factor alpha. Cancer Res 58: 1593-1598, 1998.

16. Rajeshkumar NV, Rasheed ZA, García-García E, et al: A combination of DR5 agonistic monoclonal antibody with gemcitabine targets pancreatic cancer stem cells and results in long-term disease control in human pancreatic cancer model. Mol Cancer Ther 9: 2582-2592, 2010.

17. Er E, Oliver L, Cartron PF, Juin P, Manon S and Vallette FM: Mitochondria as the target of the pro-apoptotic protein Bax. Biochim Biophys Acta 1757: 1301-1311, 2006.

18. Bevis KS, McNally LR, Sellers JC, et al: Anti-tumor activity of an anti-DR5 monoclonal antibody, TRA-8, in combination with taxane/platinum-based chemotherapy in an ovarian cancer model. Gynecol Oncol 121: 193-199, 2011.

19. Li W, Wang S, Chen $\mathrm{C}$ and Zhuang G: Induction of tumor cell apoptosis via Fas/DR5. Cell Mol Immunol 3: 467-471, 2006.

20. Ohtsuka T, Buchsbaum D, Oliver P, Makhija S, Kimberly R and Zhou T: Synergistic induction of tumor cell apoptosis by death receptor antibody and chemotherapy agent through $\mathrm{JNK} / \mathrm{p} 38$ and mitochondrial death pathway. Oncogene 22: 2034-2044, 2003.

21. Ohtsuka T and Zhou T: Bisindolylmaleimide VIII enhances DR5-mediated apoptosis through the MKK4/JNK/p38 kinase and the mitochondrial pathways. J Biol Chem 277: 29294-29303, 2002.

22. Estes JM, Oliver PG, Straughn JM Jr, et al: Efficacy of anti-death receptor 5 (DR5) antibody (TRA-8) against primary human ovarian carcinoma using a novel ex vivo tissue slice model. Gynecol Oncol 105: 291-298, 2007. 
23. Kang Z, Chen JJ, Yu Y, et al: Drozitumab, a human antibody to death receptor 5 , has potent antitumor activity against rhabdomyosarcoma with the expression of caspase- 8 predictive of response. Clin Cancer Res 17: 3181-3192, 2011.

24. Yada A, Yazawa M,Ishida S, et al: A novel humanized anti-human death receptor 5 antibody CS-1008 induces apoptosis in tumor cells without toxicity in hepatocytes. Ann Oncol 19: 1060-1067, 2008.

25. Jin H, Yang R, Ross J, et al: Cooperation of the agonistic DR5 antibody apomab with chemotherapy to inhibit orthotopic lung tumor growth and improve survival. Clin Cancer Res 14: 7733-7740, 2008.

26. Straughn JM Jr, Oliver PG, Zhou T, et al: Anti-tumor activity of TRA-8 anti-death receptor 5 (DR5) monoclonal antibody in combination with chemotherapy and radiation therapy in a cervical cancer model. Gynecol Oncol 101: 46-54, 2006.
27. Kaplan-Lefko PJ, Graves JD, Zoog SJ, et al: Conatumumab, a fully human agonist antibody to death receptor 5, induces apoptosis via caspase activation in multiple tumor types. Cancer Biol Ther 9: 618-631, 2010.

28. Kindler HL, Richards DA, Garbo LE, et al: A randomized, placebo-controlled phase 2 study of ganitumab (AMG 479) or conatumumab (AMG 655) in combination with gemcitabine in patients with metastatic pancreatic cancer. Ann Oncol 23: 2834-2842, 2012.

29. Chaudhary PM, Eby M, Jasmin A, Bookwalter A, Murray J and Hood L: Death receptor 5, a new member of the TNFR family, and DR4 induce FADD-dependent apoptosis and activate the NF-kappaB pathway. Immunity 7: 821-830, 1997.

30. Buchsbaum DJ, Zhou T, Grizzle WE, et al: Antitumor efficacy of TRA-8 anti-DR5 monoclonal antibody alone or in combination with chemotherapy and/or radiation therapy in a human breast cancer model. Clin Cancer Res 9: 3731-3741, 2003. 